Supporting information for

\title{
Approach to increase the utilization of active material in a high sulfur-loaded cathode for high areal capacity room-temperature sodium-sulfur batteries
}

Ajit Kumar,,a,b Arnab Ghosh,, ,a,c Arpita Ghosh, ${ }^{\mathrm{c}}$ Aakash Ahuja,c Maria Forsyth,,,* Douglas R. MacFarlane, ${ }^{\mathrm{b}, *}$ and Sagar Mitra ${ }^{\mathrm{c}, *}$

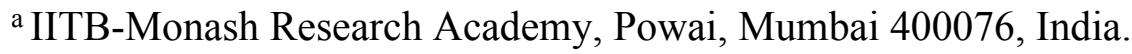

${ }^{\mathrm{b} A R C}$ Centre of Excellence for Electromaterials Science, School of Chemistry, Monash University, Clayton, Victoria 3800, Australia.

*E-mail: douglas.macfarlane@,monash.edu

'Electrochemical Energy Laboratory, Department of Energy Science and Engineering, Indian Institute of Technology Bombay, Mumbai 400076, India.

*E-mail: sagar.mitra@iitb.ac.in

${ }^{d}$ ARC Centre of Excellence for Electro materials Science, Institute for Frontier Materials and School of Engineering, Deakin University, Waurn Ponds, Geelong, Victoria 3800, Australia. *E-mail: maria.forsyth@deakin.edu.au 


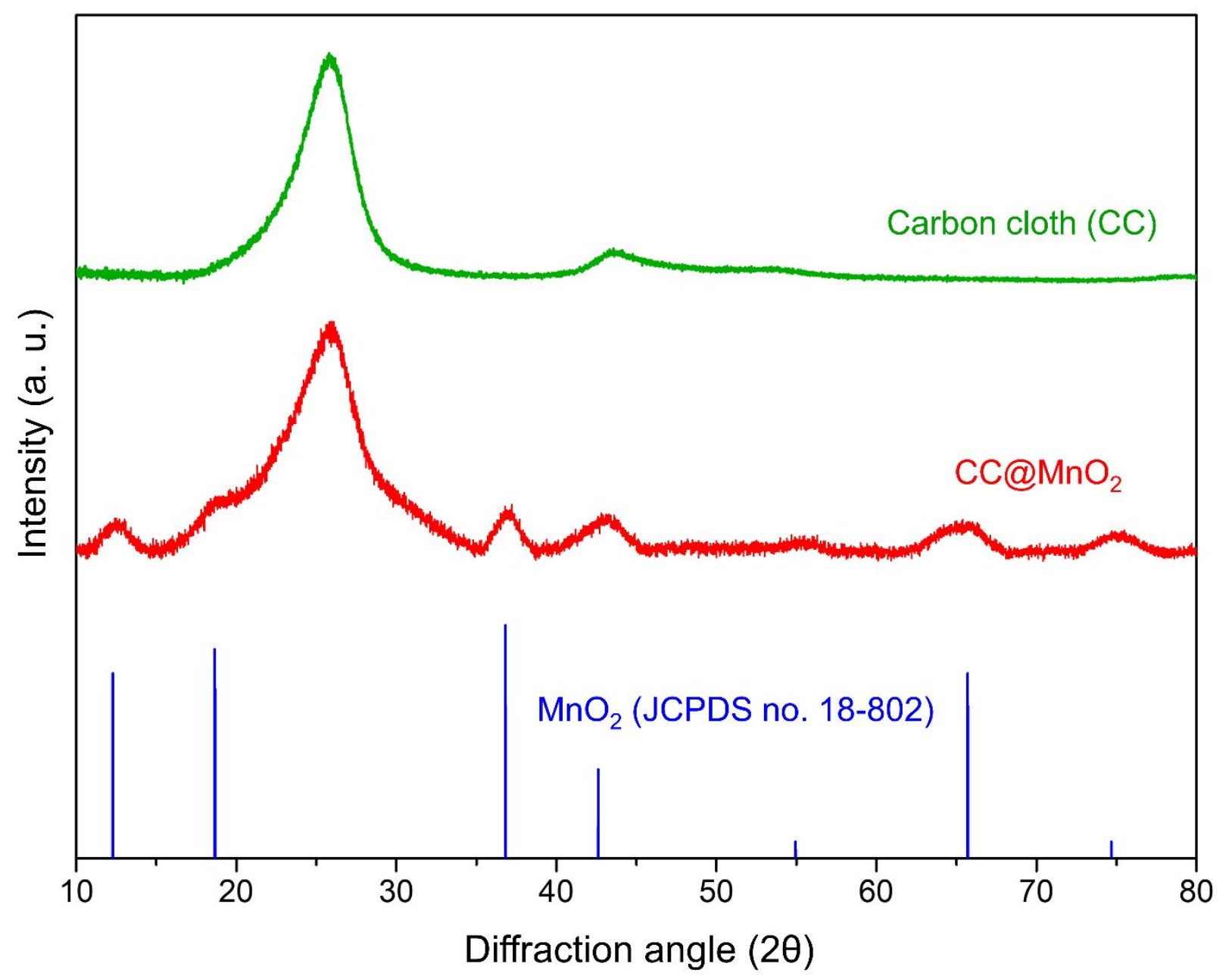

Figure S1: X-ray diffraction patterns of pristine carbon cloth and $\mathrm{MnO}_{2}$ nanoarrays decorated carbon cloth. 


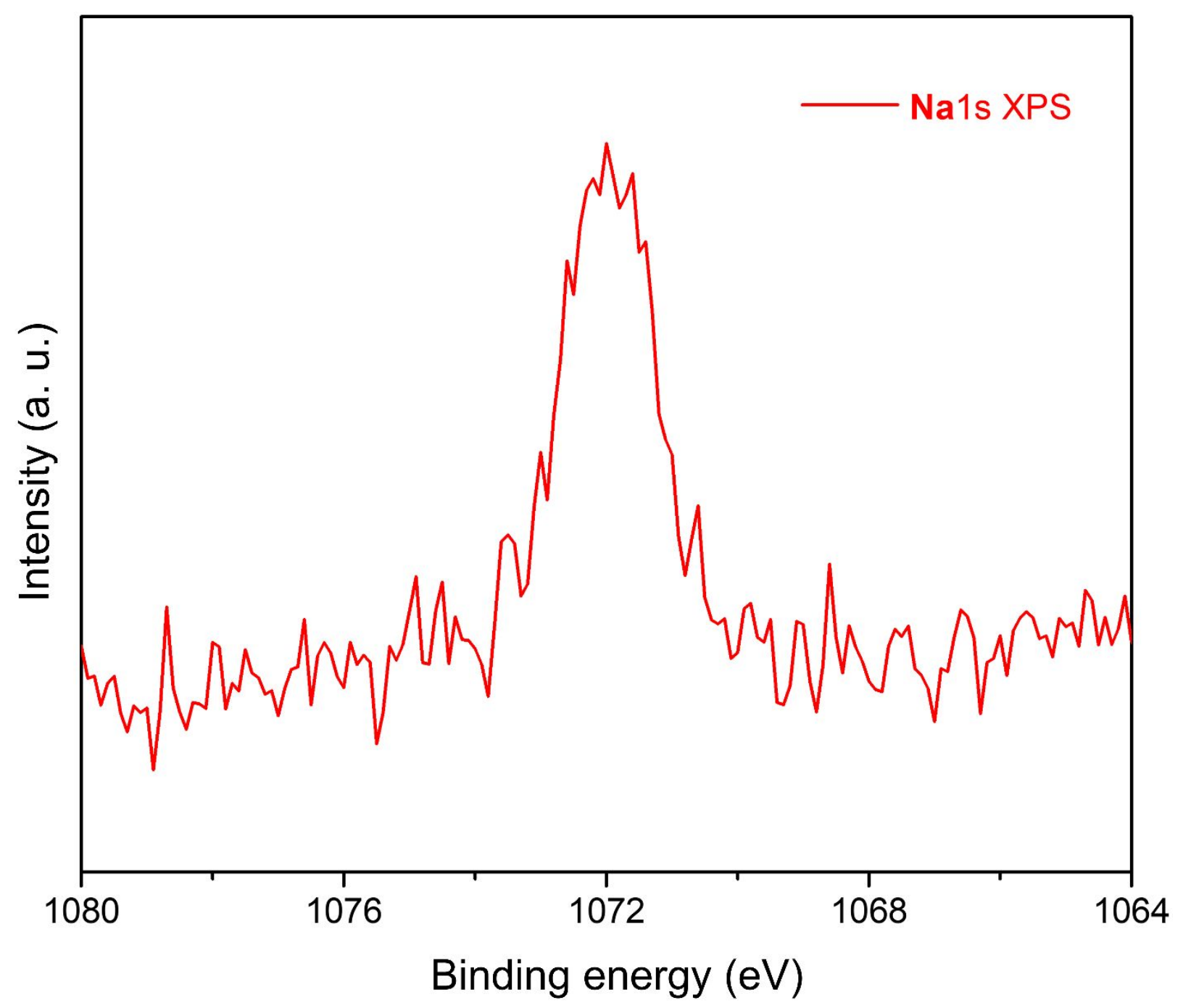

Figure S2: $\mathrm{Na}(1 \mathrm{~s})$ XPS spectrum of $\mathrm{CC} @ \mathrm{MnO}_{2} @ \mathrm{Na}-$ alg. 


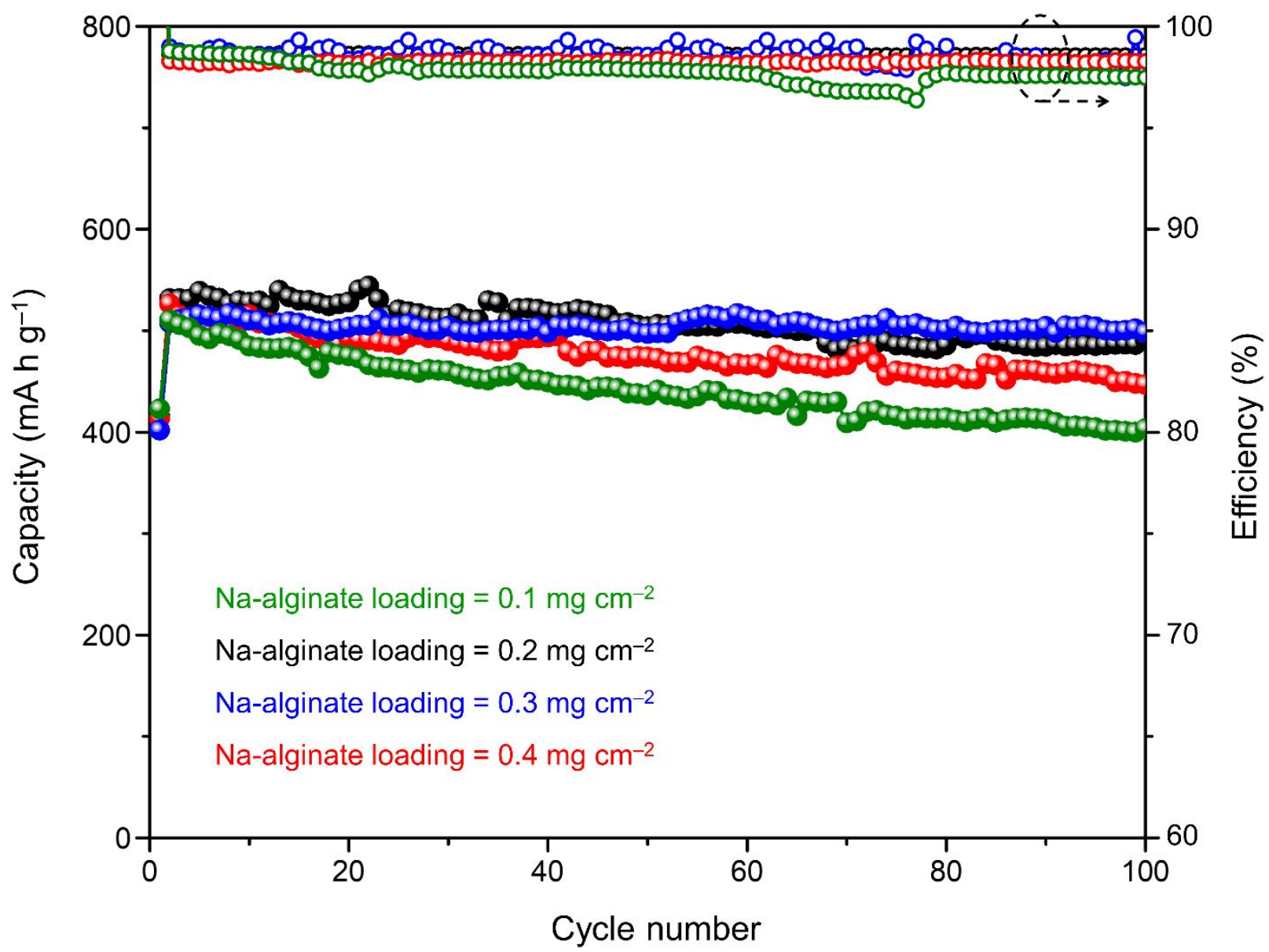

Figure S3: Cycling performance of different $\mathrm{CC} @ \mathrm{Na}_{2} \mathrm{~S}_{6} @ \mathrm{Na}-\mathrm{alg}$ cathodes (sulfur loading $1.7 \mathrm{mg}$ $\mathrm{cm}^{-2}$ ) with different areal loading of Na-alginate. 


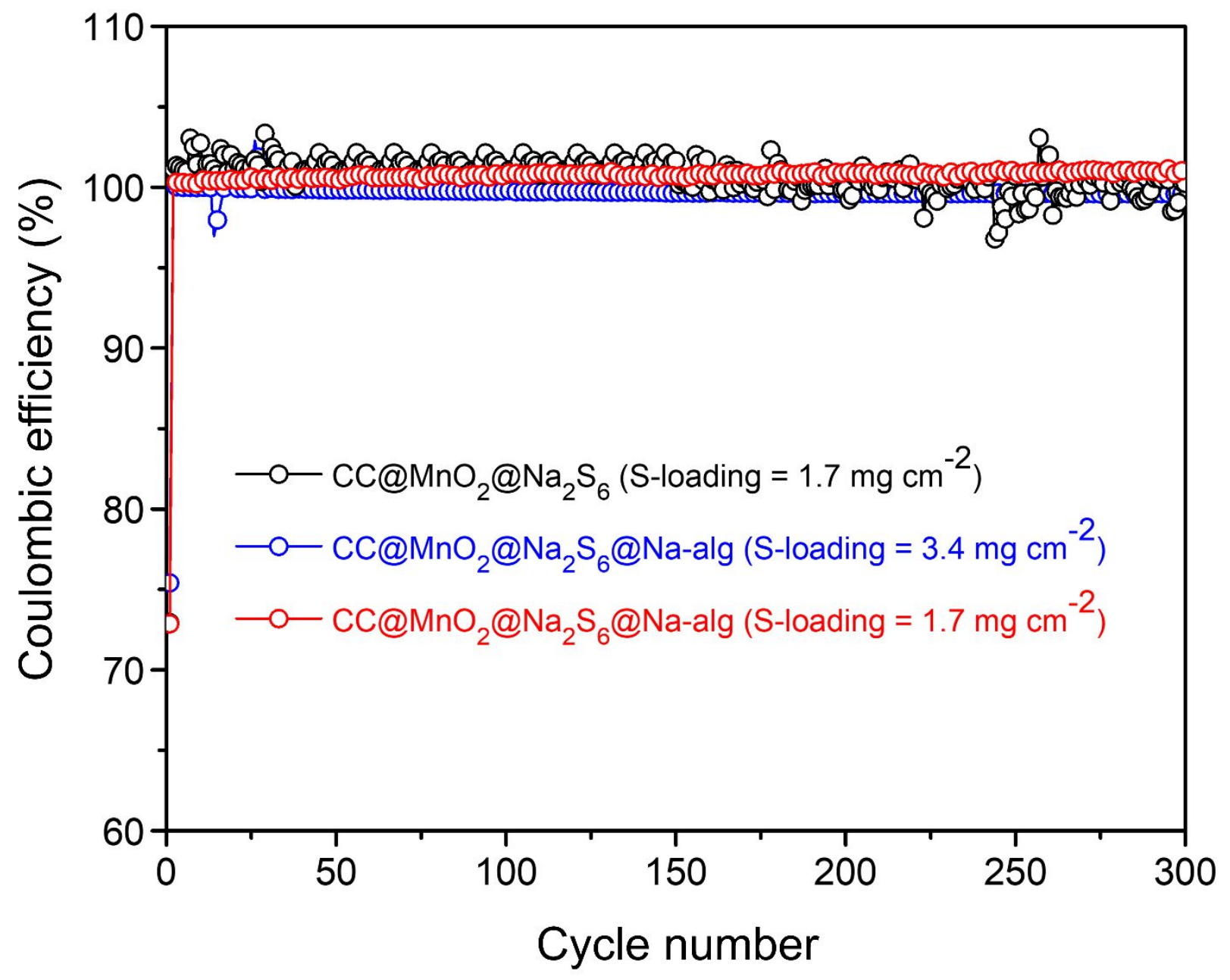

Figure S4: Coulombic efficiency vs cycle number plot at $0.2 \mathrm{C}$ for three different cathodes. 
Table S1: Comparison of relative mass of sulfur in different cathodes.

\begin{tabular}{|c|c|c|c|c|c|}
\hline $\begin{array}{l}\text { Types of current } \\
\text { collector }\end{array}$ & $\begin{array}{l}\text { Areal mass of } \\
\text { sulfur in } \\
\text { electrode }\end{array}$ & $\begin{array}{c}\text { Areal mass of } \\
\text { current collector }^{\S}\end{array}$ & $\begin{array}{c}\text { Mass of other } \\
\text { inactive components } \\
\text { in electrode }\end{array}$ & $\begin{array}{c}\text { Relative mass } \\
\text { of sulfur in } \\
\text { electrode }\end{array}$ & References \\
\hline CC@MnO & $3.4 \mathrm{mg} \mathrm{cm}^{-2}$ & $12.4 \mathrm{mg} \mathrm{cm}^{-2}$ & $0.8 \mathrm{mg} \mathrm{cm}^{-2}$ & $\sim 20.5 \mathrm{wt} \%$ & This work \\
\hline $\begin{array}{l}\text { Aluminum foil, } \\
\text { thickness }=25 \mu \mathrm{m}\end{array}$ & $0.4 \mathrm{mg} \mathrm{cm}^{-2}$ & $6.75 \mathrm{mg} \mathrm{cm}^{-2}$ & $0.43 \mathrm{mg} \mathrm{cm}^{-2}$ & $\sim 5.3 \mathrm{wt} \%$ & 6 \\
\hline $\begin{array}{l}\text { Aluminum foil, } \\
\text { thickness }=20 \mu \mathrm{m}\end{array}$ & $3.65 \mathrm{mg} \mathrm{cm}^{-2}$ & $5.4 \mathrm{mg} \mathrm{cm}^{-2}$ & $5.05 \mathrm{mg} \mathrm{cm}^{-2}$ & $\sim 25.9 \mathrm{wt} \%$ & 9 \\
\hline $\begin{array}{l}\text { Aluminum foil, } \\
\text { thickness }=20 \mu \mathrm{m}\end{array}$ & $1.1 \mathrm{mg} \mathrm{cm}^{-2}$ & $5.4 \mathrm{mg} \mathrm{cm}^{-2}$ & $3.9 \mathrm{mg} \mathrm{cm}^{-2}$ & $\sim 10.6 \mathrm{wt} \%$ & 12 \\
\hline $\begin{array}{c}\text { Copper foil, } \\
\text { thickness }=9 \mu \mathrm{m}\end{array}$ & $1.0 \mathrm{mg} \mathrm{cm}^{-2}$ & $8.06 \mathrm{mg} \mathrm{cm}^{-2}$ & $2.57 \mathrm{mg} \mathrm{cm}^{-2}$ & $\sim 8.6 \mathrm{wt} \%$ & 23 \\
\hline $\begin{array}{c}\text { Carbon paper, } \\
\text { thickness }=30 \mu \mathrm{m}\end{array}$ & $2.1 \mathrm{mg} \mathrm{cm}^{-2}$ & $3.4 \mathrm{mg} \mathrm{cm}^{-2}$ & $1.53 \mathrm{mg} \mathrm{cm}^{-2}$ & $\sim 29.9 \mathrm{wt} \%$ & 25 \\
\hline
\end{tabular}

\$areal mass of activated carbon cloth is estimated by measuring the average weight of five $1 \mathrm{~cm}^{-2}$ disks of ACC current collector prepared in different batches. The areal masses of aluminum and copper current collector of specific thickness are calculated considering the density of individual pure metal at STP.

\#other inactive components include binder, conducting carbon additive and metal oxide additives (where applicable), except current collector. 
Table S2: Comparison of relative utilization of sulfur and areal capacities in different cathodes.

\begin{tabular}{|c|c|c|c|c|}
\hline $\begin{array}{c}\text { Types of current } \\
\text { collector }\end{array}$ & $\begin{array}{l}\text { Areal mass of sulfur } \\
\text { in electrode }\end{array}$ & $\begin{array}{c}\text { \% of sulfur } \\
\text { utilization in } \\
\text { cathode }^{\dagger}\end{array}$ & $\begin{array}{l}\text { Areal capacity } \\
\text { (after } 20 \text { cycles) }\end{array}$ & References \\
\hline \multirow{2}{*}{ CC@MnO $\mathrm{Mn}_{2} @ \mathrm{Na}-\mathrm{alg}$} & \multirow{2}{*}{$3.4 \mathrm{mg} \mathrm{cm}^{-2}$} & 57.1\% (@0.2C) & $3.24 \mathrm{~mA} \mathrm{~h} \mathrm{~cm}^{-2}$ & \multirow[t]{2}{*}{ This work } \\
\hline & & 44.8\%(@1C) & $2.54 \mathrm{~mA} \mathrm{~h} \mathrm{~cm}-2$ & \\
\hline $\begin{array}{l}\text { Aluminum foil, } \\
\text { thickness }=25 \mu \mathrm{m}\end{array}$ & $0.4 \mathrm{mg} \mathrm{cm}^{-2}$ & 59.2\% (@0.1C) & $0.40 \mathrm{~mA} \mathrm{~h} \mathrm{~cm}-2$ & 6 \\
\hline $\begin{array}{l}\text { Aluminum foil, } \\
\text { thickness }=20 \mu \mathrm{m}\end{array}$ & $3.65 \mathrm{mg} \mathrm{cm}^{-2}$ & 22.6\% (@0.06C) & $1.38 \mathrm{~mA} \mathrm{~h} \mathrm{~cm}-2$ & 9 \\
\hline $\begin{array}{l}\text { Aluminum foil, } \\
\text { thickness }=20 \mu \mathrm{m}\end{array}$ & $1.1 \mathrm{mg} \mathrm{cm}^{-2}$ & 23.8\% (@0.14C) & $0.44 \mathrm{~mA} \mathrm{~h} \mathrm{~cm}-2$ & 12 \\
\hline $\begin{array}{c}\text { Copper foil, } \\
\text { thickness }=9 \mu \mathrm{m}\end{array}$ & $1.0 \mathrm{mg} \mathrm{cm}^{-2}$ & 31.8\% (@0.06C) & $0.53 \mathrm{~mA} \mathrm{~h} \mathrm{~cm}-2$ & 23 \\
\hline $\begin{array}{c}\text { Carbon paper, } \\
\text { thickness }=30 \mu \mathrm{m}\end{array}$ & $2.1 \mathrm{mg} \mathrm{cm}^{-2}$ & 51.5\% (@0.5C) & $1.81 \mathrm{~mA} \mathrm{~h} \mathrm{~cm}-2$ & 25 \\
\hline
\end{tabular}

†Percentage of sulfur utilization has been calculated considering the stable cathode capacity after 20 cycles. 

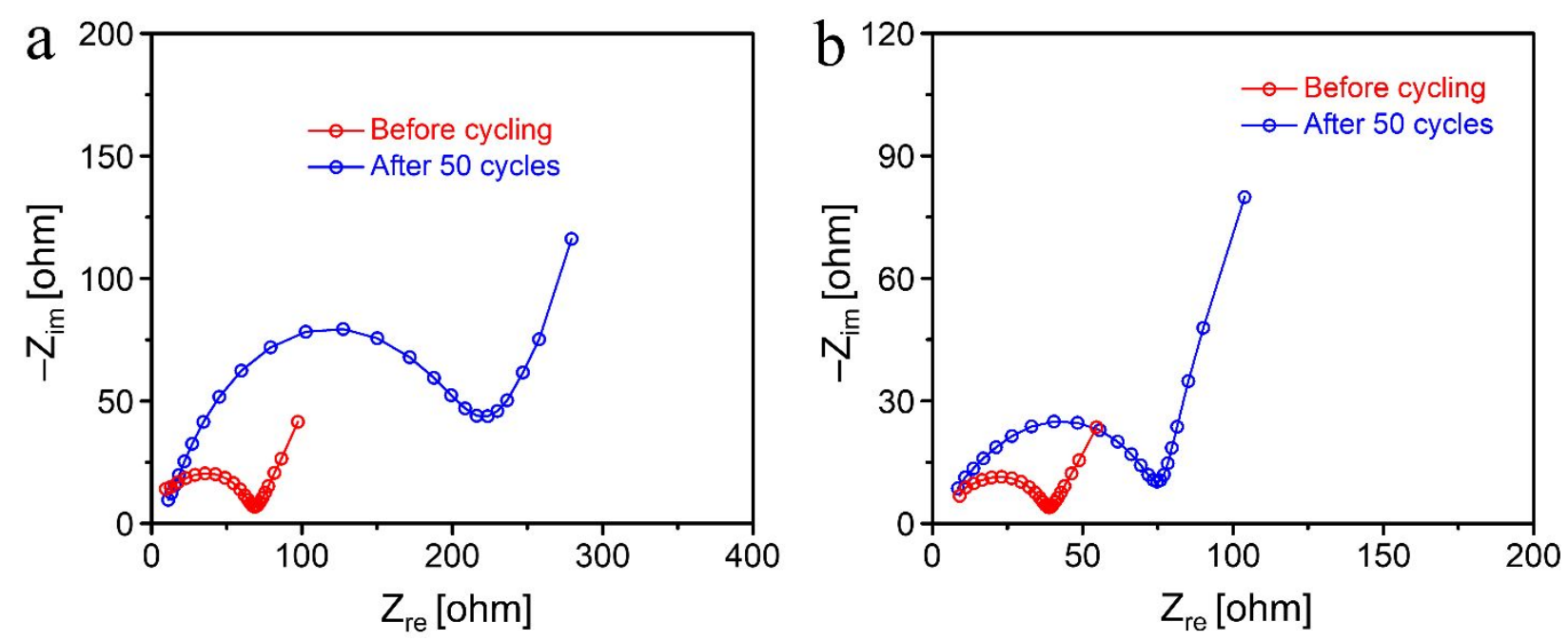

Figure S5: EIS spectra of two different RT Na-S cells containing (a) CC@ $\mathrm{MnO}_{2} @ \mathrm{Na}_{2} \mathrm{~S}_{6}$ cathode (sulfur loading=1.7 mg cm${ }^{-2}$ ) and (b) $\mathrm{CC} @ \mathrm{MnO}_{2} @ \mathrm{Na}_{2} \mathrm{~S}_{6} @ \mathrm{Na}$-alginate cathode (sulfur loading $=1.7 \mathrm{mg} \mathrm{cm}^{-2}$ ), before and after cycling. 

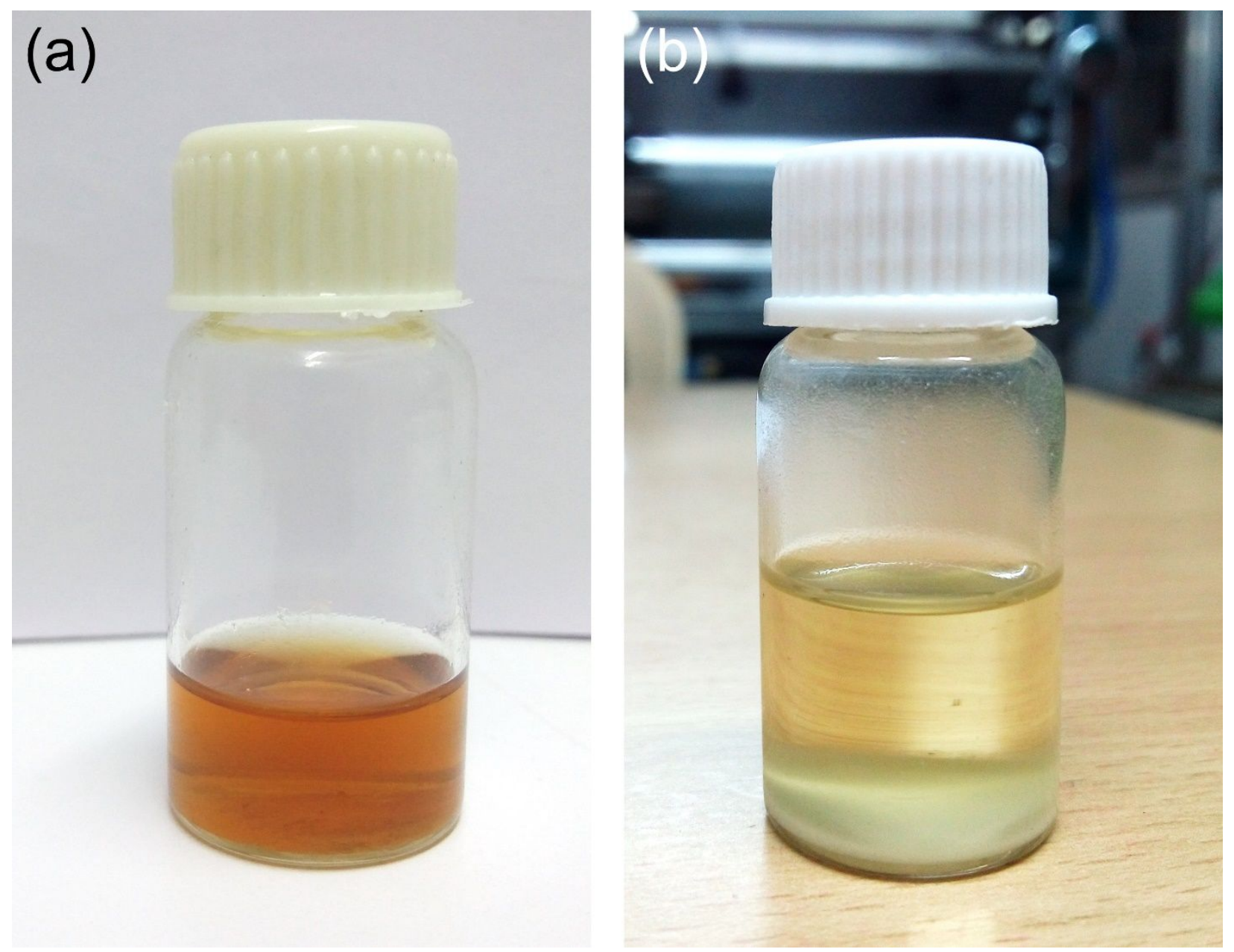

Figure S6: Digital images of $\mathrm{Na}_{2} \mathrm{~S}_{6}$ solution - (a) before addition of sodium-alginate and (b) after adding sodium-alginate. 


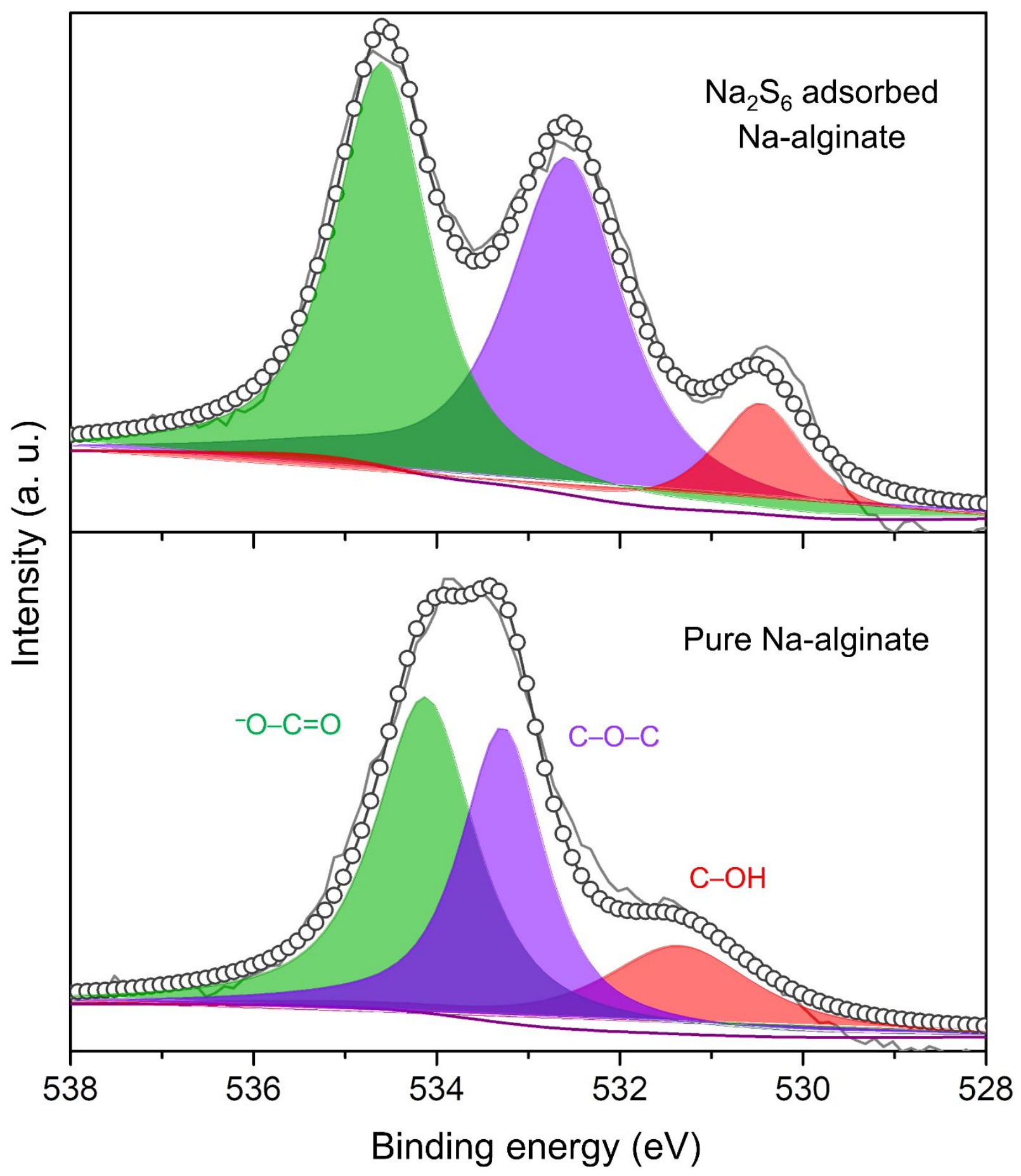

Figure S7: O1s XPS spectra of pure sodium-alginate and polysulfide-adsorbed sodium-alginate. 


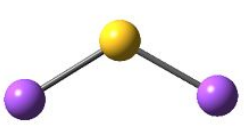

$\mathrm{Na}_{2}$

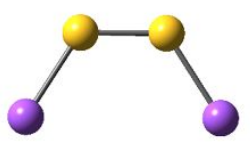

$\mathrm{Na}_{2} \mathrm{~S}_{2}$

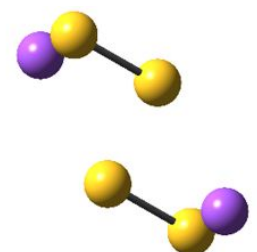

$\mathrm{Na}_{2} \mathrm{~S}_{4}$

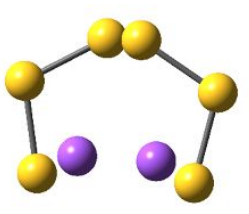

$\mathrm{Na}_{2} \mathrm{~S}_{6}$

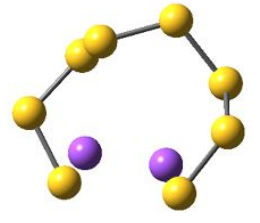

$\mathrm{Na}_{2} \mathrm{~S}_{8}$

Figure S8: Optimized structures of different sodium-polysulfides. 
Adsorption of different polysulfides on site 1
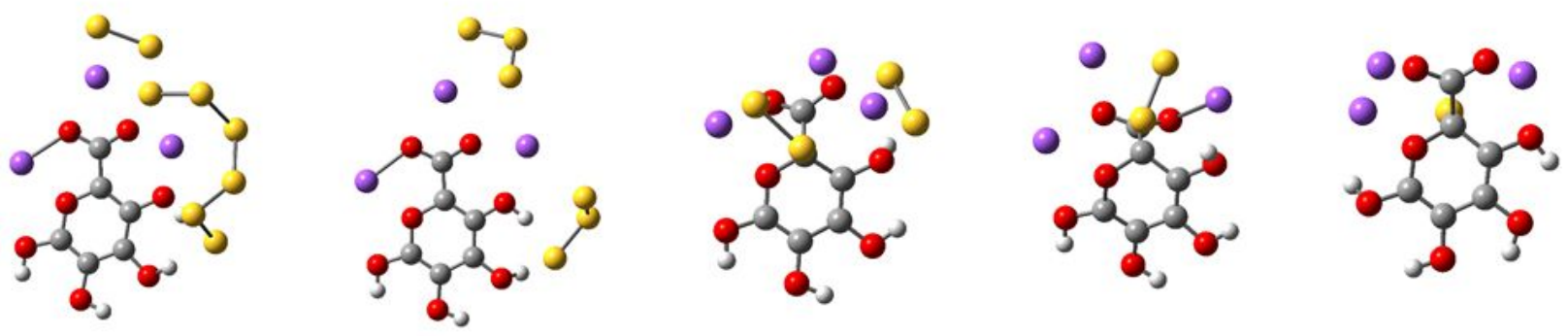

$\mathrm{Na}_{2} \mathrm{~S}_{8}$

$\mathrm{Na}_{2} \mathrm{~S}_{6}$

$\mathrm{Na}_{2} \mathrm{~S}_{4}$

$\mathrm{Na}_{2} \mathrm{~S}_{2}$

$\mathrm{Na}_{2} \mathrm{~S}$

Adsorption of different polysulfides on site 2

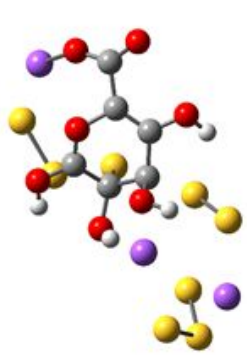

$\mathrm{Na}_{2} \mathrm{~S}_{8}$

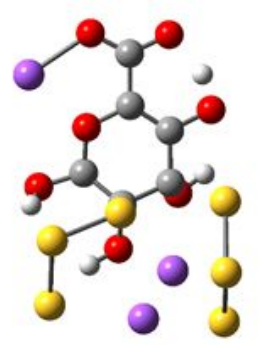

$\mathrm{Na}_{2} \mathrm{~S}_{6}$

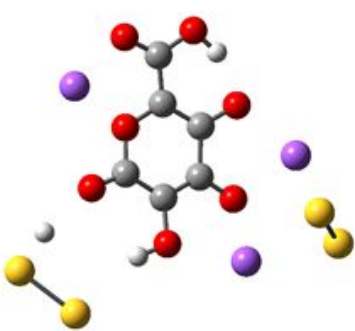

$\mathrm{Na}_{2} \mathrm{~S}_{4}$

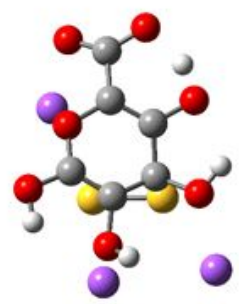

$\mathrm{Na}_{2} \mathrm{~S}_{2}$

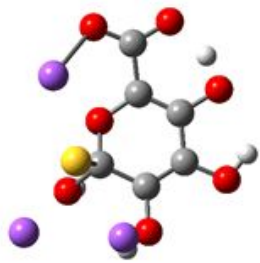

$\mathrm{Na}_{2} \mathrm{~S}$

Figure S9: Optimized geometries of different polysulfide-adsorbed systems. 

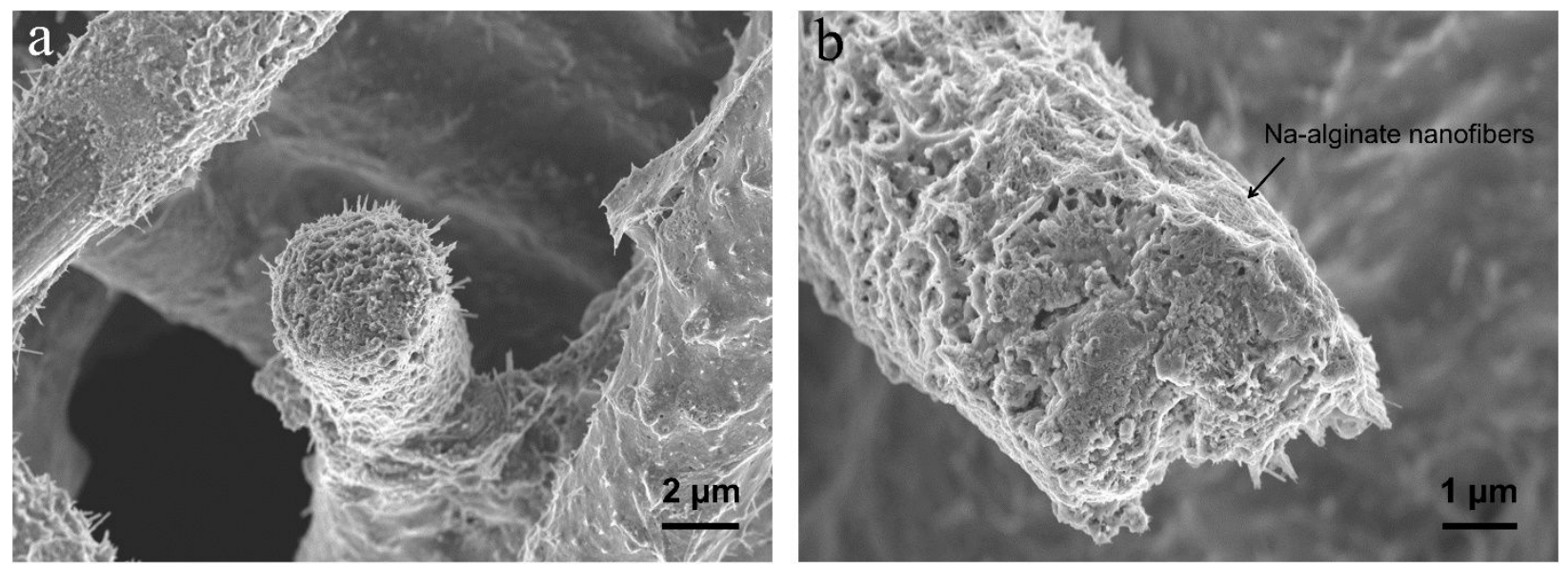

Figure S10: High-resolution cross-sectional SEM images of CC@MnO $\mathrm{Mn}_{2} \mathrm{Na}_{2} \mathrm{~S}_{6} @ \mathrm{Na}$-alg cathode after 1000 cycles. 